\title{
2D stationary resistive MHD flows: borderline to magnetic reconnection solutions
}

\author{
D.H. Nickeler ${ }^{a, *}$, H.-J. Fahr ${ }^{b}$ \\ ${ }^{a}$ Astronomical Institute, Utrecht University, Princetonplein 5, 3584 CC Utrecht, \\ the Netherlands \\ ${ }^{\mathrm{b}}$ Institut für Astrophysik und Extraterrestrische Forschung, Auf dem Hügel 71, \\ 53121 Bonn, Germany
}

\begin{abstract}
We present the basic equations for stationary, incompressible resistive MHD flows in two dimensions. This leads to a system of differential equations for two flux functions, one elliptic partial differential equation (Grad-Shafranov-like) for the magnetic flux function and one for the stream function of the flow. In these equations two potentials appear: one potential is a generalized pressure. The second potential couples the magnetic and the flow shear components of the system. With the restriction to flux or at least line conserving flows one has to solve a modified Ohm's law. For the two dimensional case these are two coupled differential equations, which represent the borderline between the resistive but flux conserving (or line conserving) case, and that of reconnective solutions. We discuss some simplified solutions of these equations.
\end{abstract}

Key words: magnetic reconnection, resistive MHD, heliopause

\section{Introduction}

A general problem of astrophysics, also reappearing in heliospheric physics, is the structure of the contact discontinuity which is located between the reverse shock $^{1}$ of a stellar wind and the counterstreaming interstellar medium. The contact discontinuity is called the heliopause or more generally the astropause.

\footnotetext{
* Corresponding author.

Email addresses: D.H.Nickeler@phys.uu.nl (D.H. Nickeler), hfahr@astro.uni-bonn.de (H.-J. Fahr).

1 In heliospheric physics this is called termination shock.
} 
Here not only two different flows are encountering, but also the electromagnetic fields transported by the flows. The aim of our work is to find magnetic field configurations in the vicinity of the stagnation point in the framework of MHD. Similar work has already been done by Priest et al. (1994). But their analysis used linearizations of the MHD-equations and their model was restricted to constant resistivities.

\section{Governing equations for stationary resistive MHD-flows in 2D}

Introducing the streaming vector $w=\sqrt{\rho} \vec{v}$ with $\vec{\nabla} \cdot \vec{v}=0$ and the electric potential $\phi_{e}(x, y)$ one can write the resistive MHD equations in the following form

$$
\begin{aligned}
\frac{\partial P}{\partial \zeta} & =\Delta \zeta \quad \frac{\partial S}{\partial \zeta}=w_{z} \quad-\frac{\partial P}{\partial \alpha}=\frac{1}{\mu_{0}} \Delta \alpha \quad-\frac{\partial S}{\partial \alpha}=\frac{1}{\mu_{0}} B_{z} \\
\frac{\partial(\zeta, \alpha)}{\partial(x, y)} & =\frac{\partial \zeta}{\partial x} \frac{\partial \alpha}{\partial y}-\frac{\partial \zeta}{\partial y} \frac{\partial \alpha}{\partial x}=\sqrt{\rho}\left(R^{z}-E_{0}\right) \\
-B_{z} w_{p} & =\sqrt{\rho}\left(R^{\zeta}+w_{p} \frac{\partial \phi_{e}}{\partial \zeta}\right) \quad \text { and } \quad w_{z} B_{p}=\sqrt{\rho}\left(R^{\alpha}+B_{p} \frac{\partial \phi_{e}}{\partial \alpha}\right)
\end{aligned}
$$

where $\vec{w}=\vec{\nabla} \zeta \times \vec{e}_{z}+w_{z} \vec{e}_{z} \equiv \vec{w}_{p}+w_{z} \vec{e}_{z}, \vec{B}=\vec{\nabla} \alpha \times \vec{e}_{z}+B_{z} \vec{e}_{z} \equiv \vec{B}_{p}+B_{z} \vec{e}_{z}$, $P \equiv p+\frac{1}{2 \mu_{0}} B_{z}^{2}+\frac{\rho}{2}\left(v_{x}^{2}+v_{y}^{2}\right)$, and $S$ is a function of $x$ and $y ; R^{\alpha}, R^{\zeta}$ and $R^{z}$ are the components of the generalized resistivity with Ohm's law given by $\vec{E}+\vec{v} \times \vec{B}=\vec{R}$, and $p$ is the plama pressure. The potentials $\zeta$ and $\alpha$ are functions of $x$ and $y$ only and are the streaming function respectively the magnetic flux function. The potential $S$ ensures the vanishing of the $z$ component of the Lorentz force, which guarantees the vanishing of the $z$ component of the pressure gradient and therefore that the equilibrium is twodimensional. Thus, the potential $S$ induces the appropriate coupling between $w_{z}$ and $B_{z}$. The equations for incompressible stationary resistive MHD in 2D had been derived by Neukirch \& Priest (1996), but without the relations for the components of the magnetic field and streaming vector in the invariant direction, which are derived in Nickeler \& Fahr (2005). Our procedure follows mainly Neukirch \& Priest (1996), however, we admit here of the $z$-component of the magnetic field and streaming vector.

To ensure the condition for magnetic field line freezing into the bulk plasma flow, one has to obey Ohm's law and the induction equation

$$
\frac{\partial \vec{B}}{\partial t}-\vec{\nabla} \times(\vec{v} \times \vec{B})=\lambda \vec{B}
$$




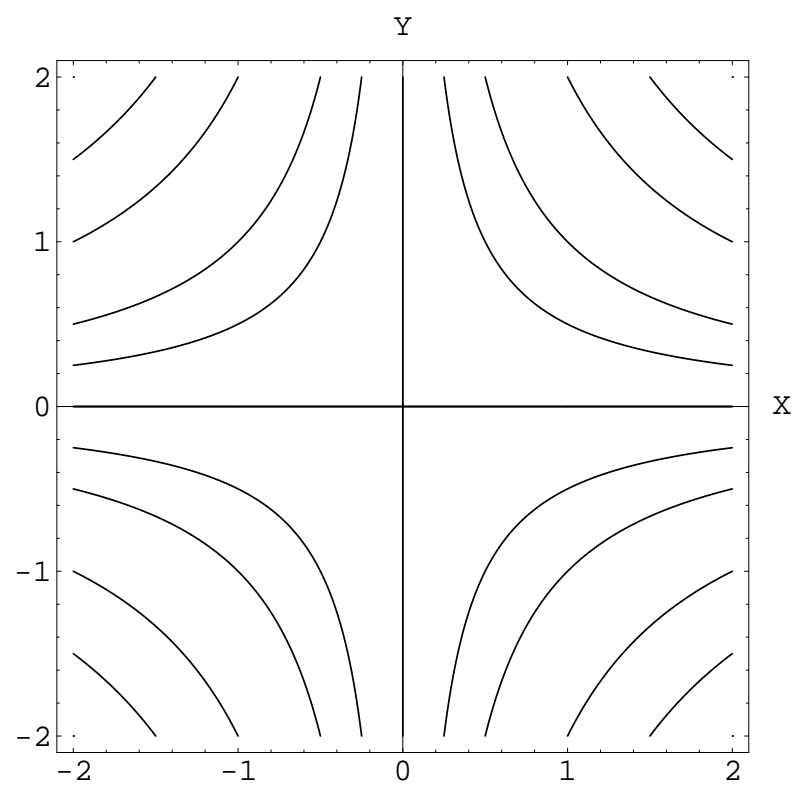

Fig. 1. Streamlines of a standard stagnation point of a symmetric astropause. The astropause is the $\mathrm{y}$-axis.

Derivations of this relation can be found in Priest \& Forbes (2000) and references therein and in Hesse \& Schindler (1988). For $\lambda=0$ this reduces to the well-known frozen-in field condition (flux conserving flow).

In the stationary two-dimensional case the above criterion must be written as

$$
\begin{aligned}
& \frac{1}{\sqrt{\rho}} \frac{\partial(\zeta, \alpha)}{\partial(x, y)}=\Lambda(\alpha) \text { and }\left(\frac{\partial B_{z}}{\partial \alpha}+\frac{\partial w_{z}}{\partial \zeta}-\frac{w_{z}}{2 \rho} \frac{d \rho}{d \zeta}\right) \Lambda(\alpha)=\Lambda^{\prime}(\alpha) B_{z} \\
\Leftrightarrow & \vec{\nabla} \times(\vec{v} \times \vec{B})=\Lambda^{\prime}(\alpha) \vec{B}
\end{aligned}
$$

for the stationary two dimensional case. Equation (6) is equivalent to the critereon for line-freezing, which is given, e.g. by Vasvliunas (1972). The term $\Lambda(\alpha)$ is the difference between the resistive term and the constant electric field $E_{0} \neq 0$ in $z$-direction ${ }^{2}$. A simple case is $\Lambda(\alpha)=$ constant $=\Lambda_{0}=-E_{0}$ which neglects resistive terms. Assuming a standard stagnation point given by the flow field $\zeta=a x y$, which is shown in Fig. 1, we are able to calculate a wide class of solutions of ideal Ohm's law for the magnetic field by using the theory of characteristics. These solutions of ideal Ohm's law are given by

$$
\alpha=-\frac{E_{0}}{2 a} \sqrt{\rho}\left(k_{1} \ln \left(x / x_{0}\right)^{2}+\left(1+k_{1}\right) \ln \left(y / y_{0}\right)^{2}\right)
$$

Field lines of a special solution of the Ohm's law around a standard stagnation point can be seen in Fig. 2. where in this special case the constants $a, x_{0}$ and $y_{0}$ $\overline{{ }^{2} E_{0}}=0$ would imply that the flow is purely field aligned 


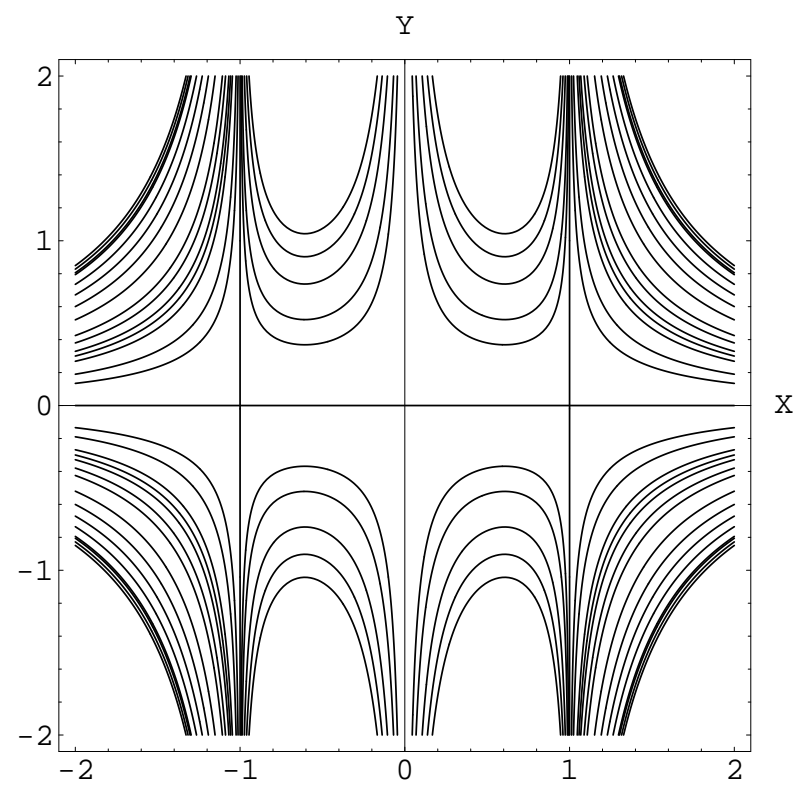

Fig. 2. Magnetic field lines resulting from solving Ohm's law with respect to the velocity field of Fig. 1 for $E_{0} \neq 0$; for $E_{0}=0$ the magnetic field would be exactly flow-aligned, if the shear components are zero.

are set to 1 and $k_{1}$ is set to -1 , which could represent the magnetic fields in the vicinity around a stagnation point of an astrosphere, where the flow vector $\vec{w}$ vanishes at the "nose" (i.e. stagnation point) of the astropause. However, in the case of ideality $(\Lambda=$ const. $)$ the velocity field diverges, although $|\vec{w}|$ remains finite, so that the "stagnation point", is only a "topological" stagnation point. Only for the field $\vec{w}$ the origin is a stagnation point. Here we have to choose the density to be zero on the separatrices to avoid magnetic singularities. Therefore around the separatrices there must be a kind of a "vacuum gap" to ensure that the mass current density vector $\sqrt{\rho} \vec{w}$ remains finite. This leads to a double separatrix structure of the magnetic field (see Fig. 2). So the constraint of "idealness" leads to a magnetic structure which is more complex then the underlying structure of the flow field.

\section{Problems and Discussion}

The class of solutions we found does not ensure that these are also solutions of the Euler equation. It seems to be more probable, that stationary MHD equilibria, conserving magnetic flux, do not exist close to the standard stagnation point, at least not without other forces, e.g. pressure anisotropies. It seems that to date only for a constant resistivity two dimensional annihilation solutions (without shear components) have been found (Neukirch \& Priest, 1996). So our ansatz gives a possibility for further investigations, to find non flux conserving, but line conserving solutions $\Lambda=\Lambda(\alpha)$, so called annihilation 
solutions, as magnetic flux is annihilated.

\section{References}

Hesse, M. \& Schindler, K., A theoretical foundation of general magnetic reconnection, J. Geophys. Res. Vol. 93 No. A6, 5559-5567, 1988

Neukirch, T. \& Priest, E., Some remarks on two-dimensional incompressible stationary reconnection, Phys. Plasmas, 3, 3188-3190, 1996

Nickeler, D.H. \& Fahr, H.-J., Reconnection at the heliopause, Adv. Space Res. 2005 (in press)

Priest, E. \& Forbes, T.: 2000, Magnetic Reconnection, (Cambridge University Press), p.233ff

Priest, E.R., Titov, V.S., Vekstein, G.E. \& Rickard, G.J., Steady linear Xpoint magnetic reconnection, J. Geophys. Res. 99, 21467-21480, 1994

Vasyliunas, V.M., Nonuniqueness of magnetic field line motion, J. Geophys. Res. 77, 6271-6274, 1972 